\title{
Túnicas Testiculares Durante la Fase de Reposo y Actividad Sexual del Conejo Macho (Oryctolagus cuniculus)
}

\author{
Morphology of Testes and it's Covers During the Phase of Rest \\ and Sexual Activity of the Male Rabbit (Oryctolagus cuniculus)
}

"Carolina Smok; ${ }^{* *}$ Estefanía Flores; ${ }^{* *}$ Gino Cattaneo; ${ }^{* * *}$ Luis Adaro \& ${ }^{* * * *}$ Mariana Rojas

SMOK, S.; FLORES, E.; CATTANEO, G.; ADARO, L. \& ROJAS, M. Túnicas testiculares durante la fase de reposo y actividad sexual del conejo macho (Oryctolagus cuniculus). Int. J. Morphol., 28(2):595-599, 2010.

RESUMEN: La domesticación del conejo (Oryctolagus cuniculus), ha permitido el paso de una reproducción de tipo anual a una de tipo no estacional, observándose pariciones durante todo el año. Sin embargo, existen parámetros reproductivos que nos indican que aún existe cierta estacionalidad, por ejemplo, la ubicación testicular, correspondiendo el conejo a un estacional de días largos. El presente estudio analiza la morfología de testículos y epidídimos, comparando la bioestructura de las cubiertas testiculares del conejo macho (Oryctolagus cuniculus), durante los meses de Enero y Septiembre. Para esto, se utilizaron seis conejos machos, tres correspondientes al mes de Enero, y tres al mes de Septiembre. Los ejemplares se encontraban clínicamente sanos y sexualmente maduros, de peso promedio $2,5 \mathrm{~kg}$. Se obtuvieron los testículos junto a sus respectivos sacos escrotales, los que se procesaron mediante técnica histológica corriente y tricrómico. Se consignó el Indice espermatogénico (IE) e índice celular interticial (ICI) según Grocock \& Clarke. Durante el mes de Enero, se evidenció una clara disminución de los parámetros reproductivos testiculares, con un IE $=3$, y un ICI=4. Por el contrario, durante el mes de Septiembre, se observó un IE=5 y ICI=5. La túnica albugínea mostró diferencias de espesor en ambos períodos. Sin embargo, se encontró un gran desarrollo de la musculatura cremastérica en ambas etapas, por lo que se concluye que ésta última no sufre atrofia durante el mes de Enero, atribuible posiblemente, a la criptorquidia facultativa que posee esta especie.

PALABRAS CLAVE: Túnica Albugínea; Saco cremastérico; Estacionalidad; Testículo.

\section{INTRODUCCIÓN}

No obstante la domesticación que ha sufrido el conejo (Oryctolagus cuniculus), y al cambio de su estatus de reproductor estacional a continuo, continúa siendo un criptórquido facultativo, una estrategia reproductiva relacionada con cambios en la ubicación testicular.

Murray (2006) describe el escroto del conejo como dos hemiescrotos que alojan los testículos, y se encuentran craneal al pene. Las cubiertas testiculares fueron descritas por Smok (2009), las cuales de superficial a profundidad se componen por: 1) dartos; 2) fascia espermática externa, constituida por varias capas de tejido conjuntivo denso; 3 ) saco cremastérico, que corresponde a una capa formada por fibras musculares de tipo estriado, muy amplia en comparación con el resto de los componentes observados; 4) fascia espermática interna, la cual es imperceptible; 5) lámina parietal de la túnica vaginal; 6) lámina visceral de la túnica vaginal, ambas separadas por la cavidad vaginal y, 7) túnica albugínea. Finalmente se ubican los testículos, los cuales presentan una posición horizontal al cuerpo.

En este trabajo, nos enfocaremos principalmente en las variaciones estacionales dentro del saco cremastérico y la túnica albugínea. En relación al saco cremastérico, Wensing (1986) describió esta estructura en la rata como un saco bilaminar, formado por dos capas de musculatura cremastérica que provienen de la prolongación de los músculos oblicuo interno y transverso del abdomen. En animales de compañía, animales de abasto y en el hombre es muy reducido (Amann \& Veeramachaneni, 2007), en cambio en

* Programa de Magíster en Cs. Biológicas, Mención Morfología, Facultad de Medicina, Universidad de Chile.

** Departamento de Cs Clínicas, Facultad de Ciencias Veterinarias y pecuarias, Universidad de Chile.

**** Departamento de Cs biológicas, Facultad de Ciencias Veterinarias y pecuarias, Universidad de Chile.

***** Laboratorio de Embriología Comparada, Programa de Anatomía y Biología del Desarrollo, Facultad de Medicina, Universidad de Chile. 
el conejo es de gran desarrollo, ya que rodea al testículo en toda su superficie (Smok). La túnica albugínea es visible sólo microscópicamente, está formada por tejido conjuntivo denso y fibras musculares lisas.

La criptorquidia facultativa que poseen los conejos, también ha sido observada por Contreras \& Bustos-Obregón (1980) en roedores caviomorfos como Octodón degus, quienes describieron que los testículos de estos animales se encuentran permanente confinados a la cavidad abdominal, descendiendo a los sacos escrotales sólo en período reproductivo. Esta interesante estrategia reproductiva relacionada con cambios temporales en la ubicación de los testículos, está controlada principalmente por el fotoperíodo, en donde se originan cambios significantes en el tamaño testicular, produciéndose la regresión testicular durante los días cortos (Boyd, 1987).

Nuestro objetivo fue describir la morfología de testículos y epidídimos; evaluar si existen cambios en la morfología microscópica del saco cremastérico y túnica albugínea, junto con observar la presencia de espermatozoides en los túbulos epididimales, comparables con las variaciones del Indice espermatogénico (IE) y células intersticiales (de Leydig) (ICI), durante la fase de reposo y actividad sexual.

\section{MATERIAL Y MÉTODO}

Se utilizaron seis conejos, provenientes del Bioterio del Instituto de Salud Pública, los cuales fueron sacrificados con una sobredosis de tiopental sódico vía endovenosa $70 \mathrm{mg} / \mathrm{Kg}$. Todos ellos eran machos clínicamente sanos y sexualmente maduros con peso corporal promedio $2,5 \mathrm{~kg}$.

Se obtuvieron los testículos, epidídimo y bolsa escrotal de 3 conejos durante el mes de Enero (10L/14O) y los otros tres durante el mes de Septiembre (12L/12O). Estas muestras se fijaron en formalina al $10 \%$, y se procesaron mediante técnica histológica corriente y tricrómico para identificar la morfología del parénquima testicular y sus cubiertas.

Se midió mediante un programa morfométrico computacional "Scalling" de Carl Zeiss, el espesor de la túnica albugínea, saco cremastérico y el diámetro de los túbulos seminíferos. Paralelamente se consignó el Indice espermatogénico (IE) según Grocock \& Clarke (1974), el cual puede ser aplicado para medir progresión de la espermatogénesis a través de la actividad del túbulo seminífero. Este índice puede ser analizado con valores que fluctúan entre el 0 y el 5, representando este último túbulos seminíferos grandes, con presencia de la línea germinal completa; mientras que el índice 0 señala túbulos seminíferos pequeños y sólo la presencia de células sustentaculares (Sertoli) y espermatogonias. Se estableció además el índice celular intersticial (ICI), con los mismos valores, en el cual índice 5 indica la presencia de muchas células intersticiales con núcleos redondos, en cambio el índice 0 se encuentran unas pocas células intersticiales aisladas. En el epidídimo se observó la presencia o ausencia de espermatozoides.

\section{RESULTADOS}

En el parénquima testicular de los conejos correspondientes al mes de Enero, se encontraron principalmente túbulos seminíferos sin lumen, con pocas espermátidas elongadas y sin espermatozoides (Fig. 1a). El diámetro tubular fue de $175 \pm 27 \mu \mathrm{m}$. Se observó un $\mathrm{IE}=3, \mathrm{y} \mathrm{ICI}=4$. Los conejos del mes de Septiembre, en cambio presentaron túbulos seminíferos con lumen, con el linaje espermatogénico completo y abundantes espermatozoides (Fig. 1b), presentando un diámetro tubular de $570 \pm 47 \mu \mathrm{m}$ y un $\mathrm{IE}=5$ e $\mathrm{ICI}=5$. Cada túbulo se encuentra limitado por una membrana basal delgada, y una monocapa de células mioides (Fig 1a). El mediastino testicular, presenta tejido conectivo denso con fibras elásticas, en donde se aprecian los túbulos que conforman la rete testis revestida por un epitelio cúbico simple. El intersticio testicular está compuesto de tejido conectivo laxo con escasos fibroblastos. También se observan vasos sanguíneos y células intersticiales.

Las cubiertas testiculares se observan en la Fig. 2. La túnica albugínea alcanza un promedio de $98 \pm 48 \mu \mathrm{m}$ en reposo y $225 \pm 110$ en actividad. Por otro lado, el saco cremastérico se mostró extraordinariamente desarrollado y visible a simple vista (Fig. 3a). Se caracterizó por estar formado por 2 capas de haces de musculatura estriada, las cuales al corte sagital, se distribuían dispuestas en sentido longitudinal y transversal (Fig. 3b). Su grosor promedio en reposo fue de $552 \pm 31 \mu \mathrm{m}$; en cambio en actividad presentó un grosor promedio de $693 \pm 42 \mu \mathrm{m}$.

El cuerpo y cola epididimaria presentó un epitelio cilíndrico seudoestratificado, con predominio de células columnares sobre las células basales mientras que hacia la cola disminuye el tamaño del epitelio, perdiéndose la diferencia de tamaños entre ambas células. Se evidenció la presencia de estereocilios en la superficie luminal, los cuales aumentan de tamaño hacia el cuerpo, siendo muy largos en esta zona, y disminuyen su longitud hacia la porción de la cola. Entre los túbulos, se aprecia una capa de células mus- 


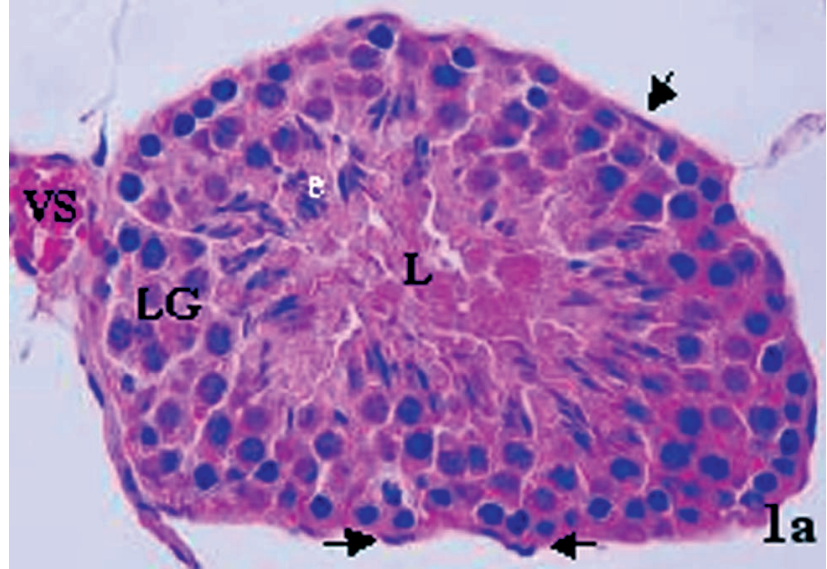

Fig. 1. (a). Mes de Enero. Túbulo seminífero. No se observa un lumen (L) propiamente tal, y la línea germinal culmina con la formación de espermátidas (e). Nótese la presencia de un vaso sanguíneo (VS), y la monocapa de células mioides (flechas). HE. 400X.

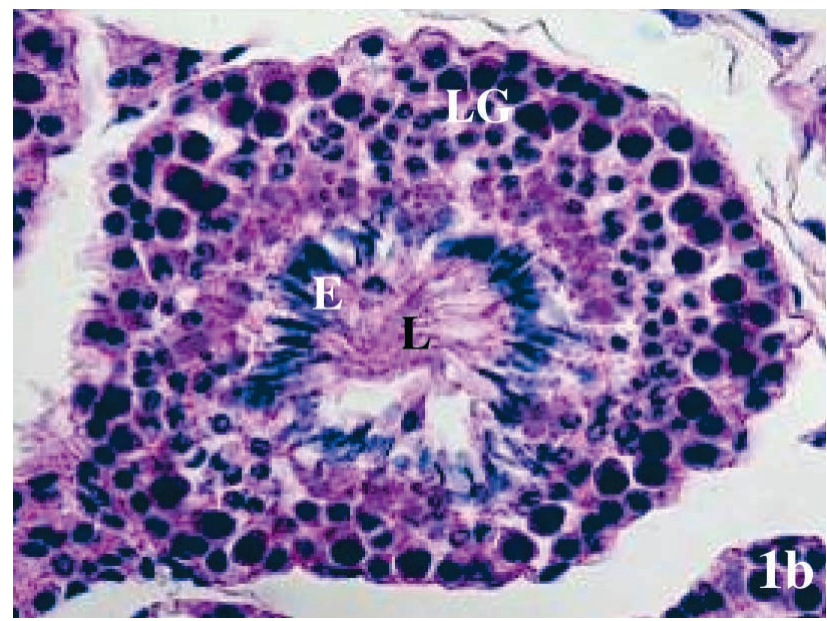

Fig. 1. (b). Mes de Septiembre. Se aprecia la línea germinal completa con la presencia de espermatozoides (E) en el lumen (L). HE. $400 X$.

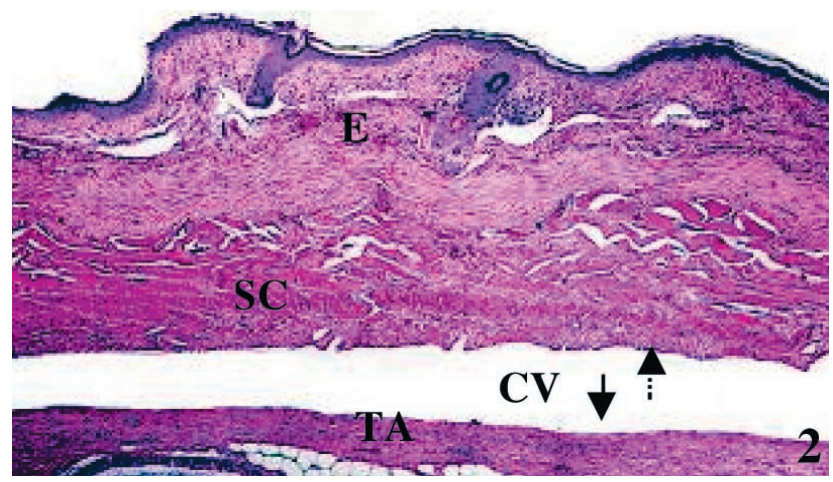

Fig. 2. Cubiertas testiculares. Se observa el tejido conectivo correspondiente al escroto (E). Bajo éste se aprecia el saco cremastérico (SC), la cavidad vaginal (CV) y la túnica albugínea (TA). La flecha indica la lámina visceral de la túnica vaginal, y la flecha punteada señala la lámina parietal de esta túnica. HE. 50X. culares lisas dispuestas en forma circular. Ésta se hace cada vez más gruesa en dirección distal y el tamaño de las fibras musculares lisas va aumentando. Además se encuentra tejido conectivo laxo (que se va tornando denso hacia caudal) y pequeños vasos sanguíneos. Durante el mes de enero, se evidenciaron espermatozoides solamente en porción de la cabeza, mientras que en el mes de Septiembre, éstos se encontraban en las tres porciones, principalmente almacenados en la cola. (Figs. 4 y 5).

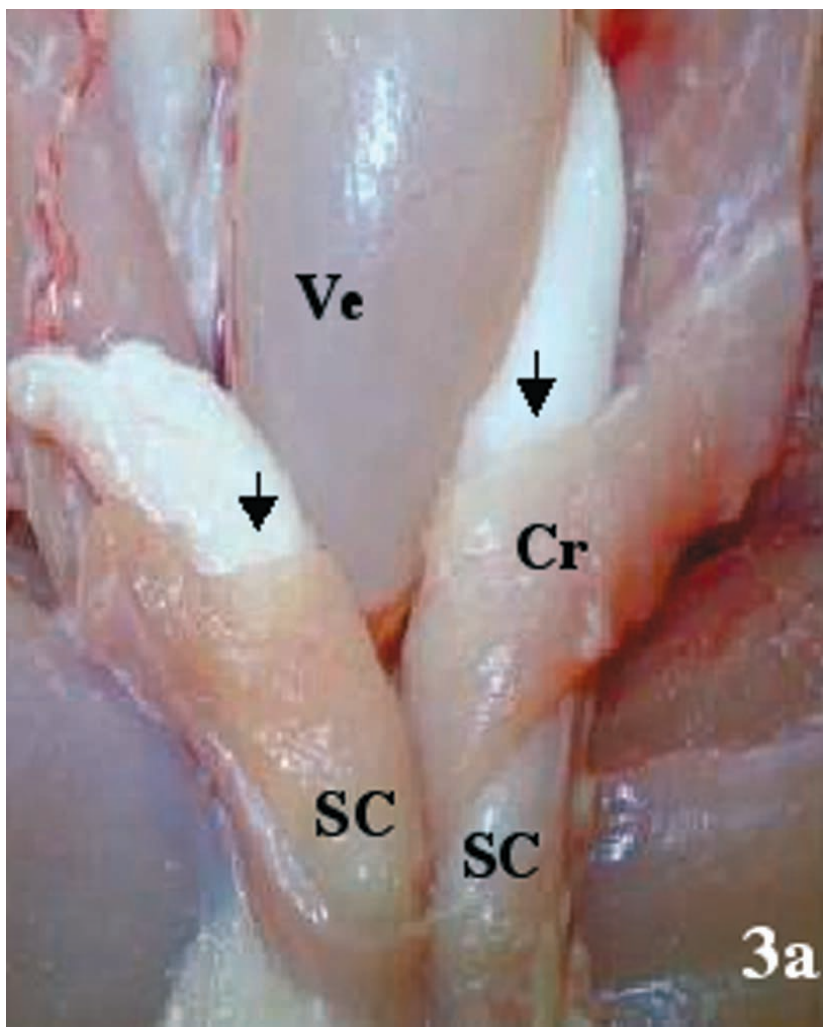

Fig. 3. (a). Se observa el músculo cremáster (Cr) y cómo se continúa de la musculatura abdominal para formar el saco cremastérico (SC). Nótese la presencia de los anillos inguinales internos (flechas) ubicados lateralmente a la vejiga (Ve).

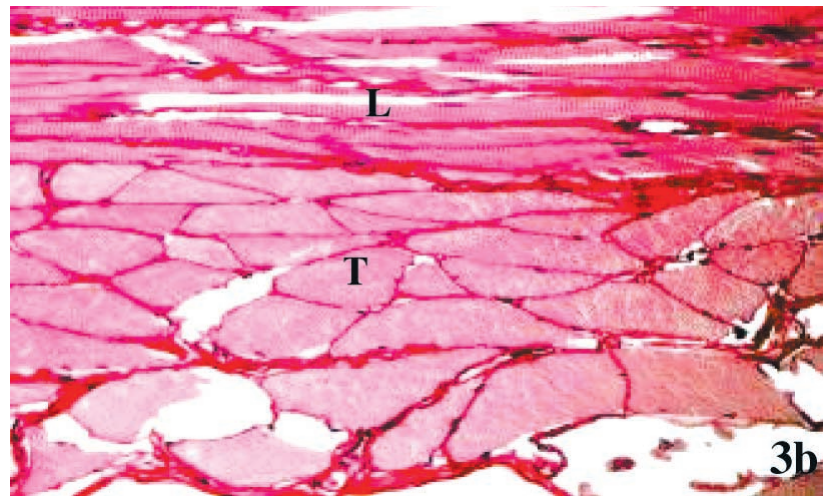

Fig. 3. (b). Corte sagital del saco cremastérico. Se observa la disposición longitudinal (L) y transversal (T) de las fibras musculares. HE. 400X. 


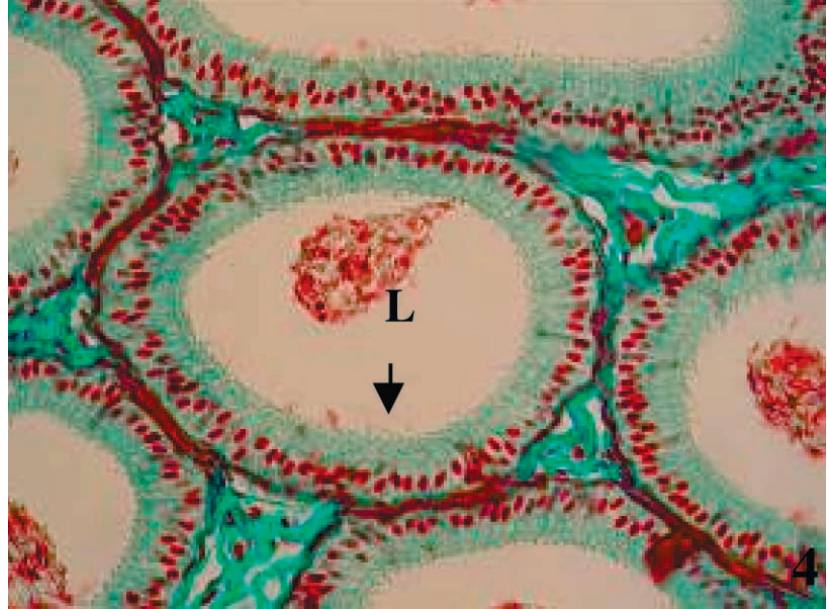

Fig. 4. Cuerpo epididimario. El lumen (L) presenta espermatozoides. Nótese la presencia de estereocilios (flechas). Los conductos se encuentran rodeados principalmente de fibras colágenas (color verde). Técnica tricrómico, 100X.

\section{DISCUSIÓN}

No obstante el conejo doméstico ha perdido su estacionalidad y se reproduce durante todo el año, se encontraron diferencias en los túbulos seminíferos. El diámetro tubular aumentó desde $175 \pm 27 \mu \mathrm{m}$ en Enero hasta $570 \pm 47$ $\mu \mathrm{m}$ en Septiembre. El ICI aumenta desde 3-4 en Enero hasta $\mathrm{ICI}=5$ en Septiembre. La única capa de células mioides observadas en la pared de los túbulos seminíferos, coincide con lo descrito por Setchell et al. (1994), quien además describe varias capas de estas células para el hombre, el carnero y el gato; por el contrario, Heyn (1987), describe para este último una sola capa.

El epitelio pseudoestratificado del cuerpo del epidídimo, junto a sus características células principales con largos estereocilios, que disminuyen de tamaño hacia la cola, en ambos períodos, han sido descritos previamente en el hamster (Calvo et al., 1997), el perro (Schimming et al., 1997) y conejo (Parra \& del Sol, 2002). Todos estos autores, junto con Setchell et al. describieron un aumento de grosor de la pared muscular que rodea el epitelio, hallazgos que concuerdan con este estudio, sin embargo, Parra \& del Sol describen para el conejo una disminución del grosor de esta musculatura en comparación con la cabeza y la cola.

Tanto conejos como roedores, a diferencia de otros animales domésticos de interés pecuario, poseen la facultad de mover libremente los testículos entre el escroto y la cavidad abdominal (Bishop, 2002), por retracción e inversión del escroto (Amann \& Veeramachaneni). Esto se produce

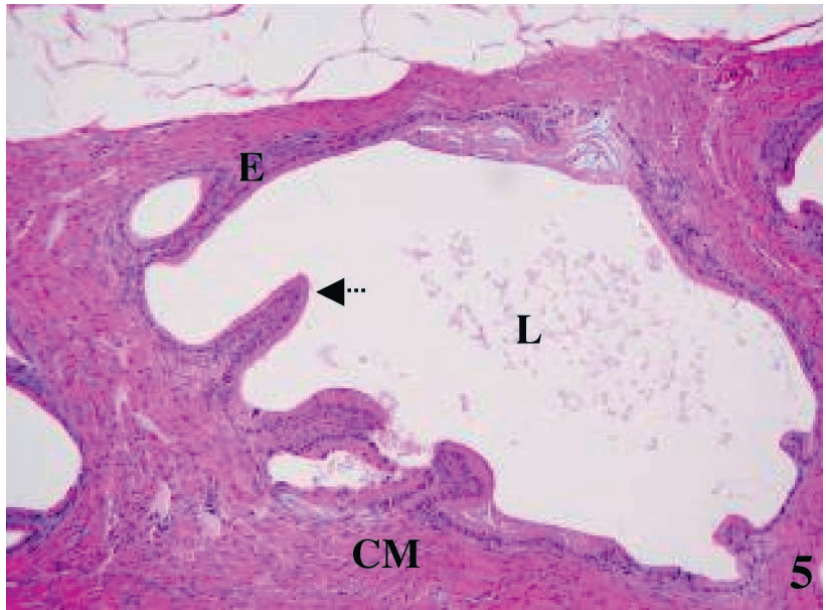

Fig. 5. Cola del epidídimo. El epitelio (E) disminuye de tamaño hacia la cola, y la capa muscular $(\mathrm{CM})$ va en aumento. El lumen es de mayor diámetro y más irregular. Nótese la disminución de tamaño de los estereocilios y la mayor densidad de éstos (flecha punteada). HE. 400X.

gracias a que son especies en la cual el anillo inguinal permanece abierto durante toda la vida (Murray). El saco cremastérico se mostró extraordinariamente desarrollado y visible a simple vista en todos los casos. Las variaciones del espesor del saco cremastérico fueron mínimas (552 \pm 31 $\mu \mathrm{m}$ en Enero hasta $693 \pm 42 \mu \mathrm{m}$ en Septiembre) pero los cambios de la túnica albugínea $(98 \pm 48 \mu \mathrm{m}$ en Enero hasta $225 \pm 110$ en Septiembre) fueron evidentes y están directamente relacionados con las variaciones de diámetro tubular y parámetros testiculares estudiados. Esta interesante adaptación de la musculatura cremastérica cuyo desarrollo se mantiene similar en los distintos períodos de actividad reproductiva, estaría relacionada con la capacidad de elevar los testículos al canal inguinal.

SMOK, S.; FLORES, E.; CATTANEO, G.; ADARO, L. \& ROJAS, M. Morphology of testes and it's covers during the phase of rest and sexual activity of the male rabbit (Oryctolagus cuniculus). Int. J. Morphol., 28(2):595-599, 2010.

SUMMARY: The domestication of the rabbit (Oryctolagus cuniculus) has allowed the passage of a reproduction of the annual rate to a non-seasonal type, observing calving throughout the year. However, there are reproductive parameters which indicate that there is still some seasonality, for example, testicular position, which corresponds the rabbit to seasonal long days. This study examines the morphology of testes and epididymis, comparing the biostructure of testicular coverings of male rabbit (Oryctolagus cuniculus) during the resting phase and sexual activity. For this, six male rabbits were used, three for the month 
of January, and three in September. The specimens were clinically healthy, sexually mature, and had an average weight of $2.5 \mathrm{~kg}$. Testes were obtained with their scrotal sacs, which are processed using current histological and trichromic technique. Spermatogenic index and interstitial cell index were recorded according to Grocock \& Clarke. During the month of January, it showed a clear decrease in testicular reproductive parameters, with $\mathrm{IE}=3$, and $\mathrm{ICI}=4$. By contrast, during the sexual activity it was observed IE $=5$ and ICI $=5$. During both periods, the albuginea tunic showed no large variations in thickness. Further, there is a great development of the cremasteric muscle in both stages, it was concluded that it does not undergo atrophy during the regression phase, possibly attributable to the voluntary cryptorchidism that this species has.

KEY WORDS: Rabbit; Albuginea tunic; Cremasteric sac; Seasonality; Testicle.

\section{REFERENCIAS BIBLIOGRÁFICAS}

Amann, R. P. \& Veeramachaneni, D. N. Cryptorchidism in common eutherian mammals. Reproduction, 133:54161, 2007.

Bishop, C. R. Reproductive medicine of rabbits and rodents. Vet. Clin. North Am. Exot. Anim. Pract., 5:507-35, 2002.

Boyd, I. L. Gonadotrophin secretion and pituitary responsiveness to LHRH in castrated and intact male rabbits exposed to different photoperiods. J. Reprod. Fert. 79:627-35, 1987.

Calvo, A; Bustos-obregón, E. \& Pastor, L. Morphological and histochemical changes in the epididymis of hamsters (Mesocricetus auratus) subjected to short photoperiod. J. Anat., 191:77-88, 1997.

Contreras, L. \& Bustos-Obregón, E. Anatomy of reproductive tract in Octodon degus Molina: A Nonscrotal rodent. Arch. Androl., 4:115-24, 1980.

Grocock, C. A. \& Clarke, J. R. Photoperiodic control of testis activity in the vole, Microtus agrestis. J. Reprod. Fert., 39:337-47, 1974.

Heyn, R. Actividad reproductiva anual del gato (Felis catus) macho: Análisis morfométrico, morfológico y hormonal. Memoria título profesional Médico Veterinario. Santiago, Chile. Universidad de Chile, Fac. Cs. Veterinarias y Pecuarias, 1987.
Murray, M. Spays and neuters in small mammals, 2006. Disponible en: http://www.ivis.org/proceedings/navc/ 2006/SAE/635.asp?LA=1

Parra, R. \& del Sol, M. Estudios histológico e histoquímico del epidídimo de conejo (Oryctolagus cuniculus). Rev. Chil. Anat., 20:269-74, 2002.

Schimming, B. C.; Vicentini, C. A.; Orsi, A. M.; Franceschini Vicentini, I. B. \& Abreu Rays, M. A. Regional Histology of the Ductus Epididymis in the Dog (Canis familiaris). Rev. Chil. Anat., 15:5-12, 1997.

Setchell, P.; Maddocks, S. \& Brooks, D. E. Anatomy, vasculature, innervation, and fluids of the male reproductive tract. In: Knobil, E. \& Neill, J. D. The Phisiology of Reproduction. $2^{\text {nd }}$ Ed. New York, Raven Press, 1994. pp.1063-175.

Smok, S. C. Descripción morfológica de testículos y estructuras asociadas de conejo (Oryctolagus cuniculus). Memoria título profesional Médico Veterinario. Santiago, Chile. Universidad de Chile, Fac. Cs. Veterinarias y Pecuarias, 2009.

Wensing, C. J. G. Testicular descent in the rat and a comparison of this process in the rat with that in the pig. Anat. Rec., 214:154-60, 1986.

Dirección para correspondencia:

Prof. Dra. Mariana Rojas

Facultad de Medicina

Universidad de Chile, Santiago

CHILE

Fono-Fax: 56-2- 2225710

Email:mrojas@med.uchile.cl

Recibido : 05-04-2010

Aceptado: 08-05-2010 
